\title{
Eventraciones, cirugía ambulatoria con anestesia local ${ }^{*}$ Ambulatory surgery using local anesthesia for incisional hernias
}

\author{
Drs. ALBERTO ACEVEDO F. ${ }^{1}$, AQUILES VITERBO S. ${ }^{1}$, JORGE BRAVO L. ${ }^{1}$, VERÓNICA DELLEPIANE L. ${ }^{1}$ \\ ${ }^{1}$ Centro de Cirugía Mayor Ambulatoria del CRS Cordillera Oriente de Santiago. Facultad de Medicina, \\ Universidad de Chile. Santiago, Chile
}

\begin{abstract}
RESUMEN
Las eventraciones constituyen la tercera causa de consulta en el Centro de Cirugía ambulatoria del CRS Cordillera, con un $11,7 \%$ y constituyen un frecuente motivo de consulta en los servicios de cirugía del país. En esta presentación damos cuenta de la cirugía ambulatoria con anestesia local de las eventraciones de tamaño pequeño y mediano. Se midieron el dolor operatorio y la satisfacción de los pacientes mediante escala visual analógica (EVA), y las complicaciones con controles efectuados a las 24 horas, una semana y un mes después de la operación. El estudio se realizó en 90 pacientes, 70 mujeres con una edad de 51 años $( \pm 12,1)$, y 20 varones con una edad de 53 años $( \pm 11,7)$ que cumplieron con los siguientes criterios: a) pacientes ASA I, y II. b) tamaño de la masa eventral inferior a $10 \mathrm{~cm}$ c) anillo eventral inferior a $3 \mathrm{~cm}$ d) ausencia de síntomas agudos abdominales e) condiciones sociales mínimas; f) edad fisiológica inferior a los 70 años y g) condición neurológica y psíquica compatible. Ni la obesidad ni la irreductibilidad de la masa herniada fueron contraindicaciones absolutas. El 66,3\% de las eventraciones tuvo un diámetro entre 5 y $10 \mathrm{~cm}$ y el $49 \%$ fue parcial o totalmente irreductible. La obesidad estuvo presente en el $56,5 \%$ de las mujeres y en el $25 \%$ de los varones. El $8 \%$ de la muestra presentó diabetes mellitus y el $28,9 \%$ hipertensión arterial compensadas. Se usó Lidocaina ${ }^{\circledR}$ alcalinizada al $0,35 \%$ en un volumen de 300 cc para infiltrar la piel y luego el espacio preperitoneal bajo la línea alba o el músculo recto en la longitud requerida. Una malla preperitoneal o retrorectal se colocó en el $22,5 \%$ de los casos. En el $10 \%$ se suturaron los bordes del anillo eventral y en el $67,5 \%$ restante se ha empleado una sutura simple de la línea media seguida de una doble sutura invaginante de la vaina de los rectos. El promedio del dolor operatorio fue 2,9 (1-8) y éste se calificó bajo 4 en el $83 \%$ de los enfermos. La satisfacción fue de 8,7 (6-10), siendo en el $98 \%$ de los casos superior a 7. Hubo un hematoma y no hubo infecciones. En esta muestra. todos los pacientes fueron autovalentes. Concluimos que las eventraciones de tamaño pequeño a mediano pueden ser intervenidas en forma ambulatoria con anestesia local en centros con experiencia en el uso de estas técnicas.
\end{abstract}

PALABRAS CLAVE: Hernia incisional, cirugía ambulatoria, anestesia local.

\section{SUMMARY}

Background: Incisional hernias account for $12 \%$ of all consultations in an outpatient hernia clinic. Aim: To report the results of ambulatory surgery performed under local anesthesia, for incisional hernias of little and middle size. Material and methods: Prospective study of patients with an American Surgical Association

*Recibido el 20 de Diciembre de 2005 y aceptado para publicación el 4 de Abril de 2006.

Correspondencia: Dr. Alberto Acevedo F.

CRS Cordillera Oriente, Las Torres 5100, Santiago.

e-mail: aacevedof @ crsoriente.cl 
risk score of I or II, a hernia size of less than $15 \mathrm{~cm}$, a hernia ring smaller than $3 \mathrm{~cm}$, absence of acute abdominal symptoms, a physiologic age under 70 years and adequate neurological conditions. Pain and satisfaction were measured by means of a visual analogical pain scale, and complications were detected in visits done 24 hours, one week and one month after surgery. Results: Seventy females aged $51 \pm 12$ years and 20 males aged $53 \pm 12$ years, were studied. Fifty six percent of females and $25 \%$ of males were obese, $8 \%$ were diabetic and $29 \%$ had high blood pressure. Sixty six percent of hernias had a sac diameter between 5 and $10 \mathrm{~cm}$ and it was partially or completely reducible in $49 \%$. A mean volume of $300 \mathrm{ml}$ of Lydocaine ${ }^{\circledR}$ $0.3 \%$ was used for local anesthesia of the skin, subcutaneous tissue and deep structures. A preperitoneal or retrorectal mesh was placed in $22.5 \%$ of the patients. A simple suture of the aponeurosis was done in $10 \%$, and a suture followed by a double invaginating suture of the rectum sheath in $67.5 \%$ of the cases. An elastic girdle was used in all patients. Mean pain score during the operation was 2.9 ( range 1-8). Satisfaction was classified with a mean score of 8.7 (range 6-10). One patient was hospitalized as a consequence of a huge wound hematoma. Pain was the main complaint, followed by light nausea that did not hinder oral feeding. All patients accomplished the self-care regime of the ambulatory program. Conclusions: Ambulatory hernia repair can be carried out in centers with experience in the management of surgery under local anesthesia.

\section{KEY WORDS: Incisional hernia, ambulatory surgery, local anesthesia.}

\section{INTRODUCCIÓN}

Entre el 1 de Enero de 1998 y el 31 de Diciembre de 2005, 169 pacientes consultaron por una eventración en el consultorio externo del CRS Cordillera Oriente. Estos pacientes eran derivados al Servicio de Cirugía del Hospital del Salvador para su tratamiento, pero comprobamos una muy difícil accesibilidad de estos pacientes al Servicio de Cirugía donde contribuyeron a conformar una larga lista de espera. Un número creciente de estos enfermos en lista de espera fueron referidos nuevamente desde los consultorios generalmente por dolor o por crecimiento en la masa eventral. El fracaso en el tratamiento de estos enfermos por cuanto el mismo grupo de cirujanos conformaba el equipo del CRS Cordillera y el equipo de pared abdominal del Servicio de Cirugía del Hospital del Salvador.

El éxito experimentado en la implementación de la cirugía ambulatoria con anestesia local de las hernias ${ }^{1}$ nos llevó a plantear la inclusión de las eventraciones pequeñas y posteriormente las de tamaño mediano en el Programa de Cirugía de las Hernias del CRS Cordillera. Los fundamentos de esta decisión no han sido económicos, sino más bien éticos y técnicos. Éticos, por cuanto era evidente que la mala accesibilidad de los pacientes a su tratamiento exponía a los enfermos a complicaciones y a un empeoramiento de su afección y técnico por cuanto el grupo quirúrgico a cargo de esta patología tanto en el SSMO cono en el Servicio de Cirugía del Hospital del Salvador tenía la capacitación suficiente. El análisis de la literatura nos permitió comprobar que la anestesia local se utiliza en algunos centros especializados para el tratamiento de las hernias umbilicales, epigástricas y de las eventraciones de tamaño pequeño ${ }^{2-5}$.

Durante el período de tiempo que abarca este estudio 109 pacientes constituyendo el $64,5 \%$ del total de los pacientes derivados desde los consultorios por una eventración fueron clasificadas como pequeñas o medianas y de ellos 90 reunieron las condiciones para ser incorporados al proyecto de manejo ambulatorio con anestesia local. En la presente comunicación damos cuenta de nuestros resultados

\section{MATERIAL Y MÉTODO}

La evaluación preoperatoria de los enfermos se efectuó en el consultorio externo según técnicas estándar de los pacientes a ser intervenidos con anestesia local

El diámetro de las eventraciones lo determinó el cirujano al momento de realizar el examen físico utilizando una regla. El diámetro del anillo se determina introduciendo los dedos en éste previa reducción de la masa eventral. En las eventraciones de la línea media la separación de los rectos se determina con el canto de la mano tras reducción de la masa eventral y solicitando al enfermo que levante la cabeza y el tronco para poner tensos los músculos.

El presente estudio se realizó en 90 pacientes que cumplieron con las condiciones para ser intervenidos con esta modalidad y firmaron el consentimiento informado. Se trató de 70 mujeres con una edad promedio de $51 \pm 12,1$ años y 20 varones con una edad de $53 \pm 11,7$ años. 
Las condiciones establecidas para acceder al programa fueron una edad fisiológica inferior a los 70 años; encontrarse en los criterios 1 y 2 de la ASA; una masa eventral única o múltiple inferior a los $10 \mathrm{~cm}$ de diámetro; un anillo o una separación de los bordes de los rectos inferior a los $3 \mathrm{~cm}$; ausencia de síntomas agudos; condiciones familiares y de vivienda compatibles con el cuidado postoperatorio en el domicilio, y condiciones aceptables psíquicas y neurológicas. La irreductibilidad total o parcial no fue una contraindicación operatoria.

EI IMC fue superior a 30 en el $56,5 \%$ de las mujeres y en el $25 \%$ de los varones y superior a 40 en el $7 \%$ de las mujeres y en el $5 \%$ de los varones, no constituyendo una contraindicación operatoria. La diabetes mellitus se presentó en el $8 \%$ de la muestra siendo insulino dependiente en 1 caso. El $28 \%$ de la muestra se encontraba en tratamiento por una hipertensión arterial. Los pacientes ASA 2 fueron derivados al especialista para su pase operatorio y una compensación de los valores de glicemia se consideró indispensable en los diabéticos.

Una vez decidida la intervención los pacientes fueron derivados a la Unidad de Pabellón donde fueron entrevistados por la enfermera quien instruyó al paciente y a un familiar sobre la técnica anestésica a utilizar, le entregó las indicaciones pre y postoperatorias, fijó la fecha probable de la intervención y obtuvo el consentimiento informado. Los pacientes recibieron un informativo y sus indicaciones impresas.

El día de la intervención los pacientes concurrieron habiendo ingerido sólo líquidos y sin interrupción de sus tratamientos farmacológicos. Se usó Amidona diluida por vía iv en bolos de $20 \mathrm{mg}$ durante la intervención y quimioprofilaxis en los diabéticos, en procedimientos que por su complejidad requerían de una gran disección de planos anatómicos y en los casos de una cirugía prolongada.

La anestesia local se efectuó en forma monitorizada con la participación de una enfermera, la que confeccionó una ficha anestésica conteniendo los valores tensionales, el pulso, la respiración y la oximetría. Igualmente se monitorizó la actividad cardíaca en un cardiógrafo.

La anestesia local se efectuó con un volumen total de $300 \mathrm{ml}$ de Lidocaina al 0,35\% mediante infiltración por planos. Primero la dermis y el tejido celular subcutáneo y tras incidir estos planos, el plano aponeurótico y/o de los músculos rectos.

Inicialmente realizamos incisiones circunscritas a la masa eventral, pero tras comprobar en 2002 la multisacularidad de las eventraciones ${ }^{6}$ efectuamos rutinariamente la resección de un losanjo de la piel y expusimos la aponeurosis en la extensión de la cicatriz operatoria. En los primeros casos procuramos poner en todas las eventraciones con un anillo superior a $1,5 \mathrm{~cm}$ una malla preperitoneal lo que efectuamos en 20 oportunidades. En 7 pacientes con eventraciones pequeñas efectuamos una sutura corrida de los bordes aponeuróticos con Ethybond ${ }^{\circledR}$ y con una relación longitud del hilo/longitud de la herida de 4 , según lo recomendado por Israelsson ${ }^{7}$, y en 2 casos realizamos un técnica de Mayo. En los restantes 60 pacientes, todos ellos con eventraciones de la línea media, tras liberación de las adherencias de los sacos al anillo aponeurótico (desvirtualización) y reducción de éstos realizamos una reparación mediante sutura corrida de $\mathrm{Vycril}^{\circledR}$ 2-0 seguida de una doble sutura invaginante de la vaina de los rectos con Ethybond 8 . Suturamos el tejido celular subcutáneo con puntos separados de $\mathrm{Vycril}^{\circledR} 0$ anclados a la aponeurosis lo que nos permitió prescindir de un drenaje.

Una vez completada la intervención el paciente deambuló, asistido por la enfermera hasta la sala de recuperación vecina donde reposó hasta el alta tras un período de observación de 60 a 120 minutos. La investigación del dolor y de la satisfacción del paciente la efectuó la enfermera mediante EVA de 10 puntos.

Los controles se efectuaron por el equipo quirúrgico al día siguiente, al séptimo y al trigésimo día después de la intervención ciñéndose a una ficha preimpresa. La información se agregó a la base de datos del proyecto de hernias elaborada con el programa de cálculo epidemiológico Epi Info 2000 que permite el manejo estadístico de la información.

\section{RESULTADOS}

En todos los casos las intervenciones pudieron realizarse sin incidentes. El promedio del dolor operatorio fue de $2,9(1-8)$ y el $83 \%$ de los pacientes refirió un dolor inferior a 4 . La satisfacción fue calificada con un puntaje promedio de 8,7 (6-10) con el $98 \%$ de los pacientes calificando la satisfacción con un puntaje superior a 7 .

Una enferma se mantuvo en observación durante 6 horas por una hipotensión durante la intervención. El total de los enfermos asistió al primer control al día siguiente de la intervención consignándose un hematoma que obligó a la hospitalización del paciente y a un manejo quirúrgico. El 12\% de los casos refirió nauseas durante las primeras horas después de la operación, transitorias y que 
no impidieron la ingestión de líquidos y alimentos. El dolor postoperatorio fue la principal molestia referida por los enfermos. Éste fue referido por los enfermos en su control efectuado en la primera semana, persistió durante 3 o 4 días y fue calificado con un puntaje promedio de la EVA de 4,2 (2-8). El dolor no impidió que los pacientes fueran autovalentes durante todo el período postoperatorio.

El examen de la herida efectuado el primer día permitió comprobar un hematoma de considerable volumen que requirió de la hospitalización del paciente. Éste se manejó en forma conservadora. No se consignaron infecciones en este grupo de pacientes durante el seguimiento de 30 días, considerando como tal a la salida de pus en forma espontánea o tras incisión quirúrgica.

\section{DISCUSION}

El manejo de las eventraciones de la línea media depende del tamaño de la masa eventral y el diámetro del anillo. Estos factores determinarán la técnica quirúrgica a utilizar en cada caso y que va desde la simple sutura en un plano a las plastías músculo aponeuróticas tipo Albanese ${ }^{9}$. Courtney y cols, en un estudio reciente ${ }^{10}$ destaca la ausencia de normas claras de cuando y cómo reparar una eventración. Si bien el propósito de esta comunicación no es resolver este problema estimamos que una clasificación de las eventraciones nos permitirá precisar de mejor forma en quién efectuar procedimientos ambulatorios con anestesia local.

Mientras Ponka ${ }^{11}$ clasifica a las eventraciones según la masa herniaria, el criterio utilizado generalmente es el diámetro del anillo. Jessen y Soerensen ${ }^{12}$ consideran pequeñas a las eventraciones con anillos de menos de $2 \mathrm{~cm}$, medianas entre 2 y $5 \mathrm{~cm}$ y grande cuando éste excede los $5 \mathrm{~cm}$. Herszage ${ }^{13}$ considera pequeñas las eventraciones cuyo anillo es inferior a $4 \mathrm{~cm}$, y Schumpelick ${ }^{5}$ presenta una clasificación donde considera pequeñas a las eventraciones con anillos bajo 2 $\mathrm{cm}$, moderadas a aquellas entre 2 y $4 \mathrm{~cm}$ y grandes a aquellas con diámetros mayores.

Con criterio meramente pragmático y orientado a normar la técnica quirúrgica a utilizar en nuestros pacientes, llamamos pequeñas a las eventraciones de la línea media cuando los borde aponeuróticos se pueden afrontar sin tensión con una simple sutura y ello se observa en eventraciones con un anillo de un diámetro inferior a $1,5 \mathrm{~cm}$ (el grosor del dedo índice); llamamos medianas a la eventraciones cuyos bordes se separan hasta $3 \mathrm{~cm}$ (el grosor de dos dedos) y que por afrontarse con alguna tensión requieren un tipo de sutura más compleja, como la de $\mathrm{Mayo}^{14}$ o la sutura simple asociada a doble sutura invaginante que presentamos en esta oportunidad; llamamos grandes a las eventraciones con anillos entre 3 y $7 \mathrm{~cm}$ (el grosor de todos los dedos de la mano sin el pulgar) y que requieren de técnicas especiales como la de WeltiEudel (citado por Barroetaveña) o la colocación de una prótesis retrorrectal ${ }^{15}$. Finalmente llamamos muy grandes a las eventraciones con anillos o con una separación de los planos aponeuróticos superior a los $7 \mathrm{~cm}$ y que requieren de plastías músculo aponeuróticas como la técnica de Albanese ${ }^{9}$ o la técnica de separación de componentes ${ }^{16}$. Si bien en Chile se ha efectuado algunas publicaciones sobre el tratamiento de las eventraciones, no se han dado a conocer experiencias con el uso de la anestesia local.

Como en otras materias, en la cirugía mayor ambulatoria (CMA) con anestesia local se ha abierto un amplio campo de experimentación clínica de la mano con la experiencia del grupo y del desarrollo tecnológico. El presente estudio traduce este desarrollo en el Centro de CMA del CRS Cordillera.

En nuestro policlínico de hernias el $11,7 \%$ de las consultas es motivada por una eventración y aproximadamente un $70 \%$ de ellas son de tamaño pequeño o mediano bastante similar a las hernias umbilicales y epigástricas de diversa complejidad que operamos rutinariamente. Diversos autores ${ }^{2-4}$ destacan que la anestesia local es la anestesia de elección en las hernias umbilicales y epigástricas y Schumpelick ${ }^{5}$ la recomienda también en eventraciones de tamaño pequeño.

Con estos antecedentes decidimos iniciar nuestra experiencia con esta patología, contribuyendo a resolver el problema del gran volumen de pacientes con eventraciones de tamaño mediano que esperan largo tiempo para ser operados en hospitales de alta complejidad, especialmente si son obesos, diabéticos o hipertensos, circunstancias que aumentan el riesgo del uso de la anestesia general o espinal.

Eso es de especial importancia si se considera que el $43 \%$ de los pacientes de nuestra serie eran obesos con un IMC superior a 30 .

Una buena técnica y experiencia en el uso de la anestesia local son esenciales para efectuar la cirugía de las eventraciones en las que se realiza la extirpación de la cicatriz y la revisión del plano aponeurótico en toda su extensión. En estas circunstancias, tanto la apreciación del dolor operatorio como de la satisfacción operatoria muestran valores similares a los observados en las herniorrafias inguinales complejas. De especial interés nos parece la disminución del dolor apreciada al susti- 
tuir o complementar la infiltración anestésica preperitoneal, con la del músculo recto dentro de su vaina en toda la longitud del defecto ${ }^{17}$.

Las complicaciones postoperatorias precoces fueron las habituales en la cirugía electiva de la pared abdominal, no apreciándose infecciones de la herida en esta muestra y consignando sólo un hematoma. El dolor durante los primeros días del postoperatorio es el mayor problema de la cirugía de las eventraciones de la línea media. Esto se aprecia también en los pacientes ambulatorios ello debe ser mencionado en la entrevista preoperatorio al momento de conseguir el consentimiento informado.

Concluimos que las técnicas acá propuestas para el tratamiento de las eventraciones de tamaño pequeño y mediano. El desarrollo de centros de cirugía ambulatoria con anestesia local debe contribuir a descongestionar los centros terciarios de salud y hospitales de alta complejidad y por lo tanto a disminuir las listas de espera.

\section{REFERENCIAS}

1. Acevedo A, Gallego A. Cirugía mayor ambulatoria de las hernias. Experiencia de 5 años en el CRS Cordillera Oriente de la ciudad de Santiago. Rev Chil Cir. 2004; 56: 166-171

2. Muschaweck U. Umbilical Hernia. Abstract book of the 2nd Internacional Hernia Congress, London, 2003: S7.8.

3. Kurzer M, Belsham P A, Kark A E. Tension-free mesh repair of umbilical hernia al a day case using local anesthesia. Hernia. 2004; 8: 104-107.

4. Arroyo Sebastián A, Pérez F, Serrano P, Costa D, Oliver I, et al. Is prosthetic umbilical hernia repair good to replace primary herniorraphy in the adult patient? Hernia, 2002; 6: 175-177.

5. Schumpelick, V. Hernien. G. Thiem Verlag, Stuttgart, 2000: 266-297
6. García A, Acevedo A, Bravo M. Estudio morfológico de las eventraciones de la línea media. Libro de Resúmenes del LXXVII Congreso Chileno de Cirugía, 2004: 130

7. Israelsson I A, Johnson T, Knutsson A. Suture technique and healing in midline laparotomy incisions. Eur J Surg 1996; 162: 605-609.

8. Acevedo A, Viterbo A, Bravo J, Dellepiane V. Eventrorrafia ambulatoria con anestesia local mediante técnica de doble sutura invaginante isotensional. Rev Argent de Cir, en prensa.

9. Albanese AR. Remodelación por movilización músculo-aponeurótica en el tratamiento de las grandes eventraciones del abdomen. Dia Méd 1976; 12: 418429

10. Courtney C, Lee AC, Wilson C, O Dwyer P J. Ventral hernia repair: a study of current praxis. Hernia 2003; 7: 44-46.

11. Ponka $\mathrm{J}$ L. Hernias of the abdominal wall. W $B$ Saunders Co, Phyladelphia, 1980 369-396.

12. Jenssen C, Sorensen B M. Incisional hernia. Acta Chir Scand 1967; 133: 487-501

13. Barroetaveña J, Herszage L, Tibaudin HA, Barroetaveña JL, Ahualli CE. Cirugía de las eventraciones. Editorial El Ateneo 1988: 95-153

14. Mayo W J. Radical cure of umbilical hernia. Ann Surg 1899; 34: 276-282.

15. Rives J .Pire J C, Falment J B. Traitement des eventrations. Encycl Med Chir Paris, 1977; 4.0.07: 40-165.

16. Ramirez OM, Girotto JA. Clousure of chronic abdominal wall defects: The component separation technique. En Bendavid R, Abrahamson J, Arregui M E, Flament J B, Phillips E H. Abdominal wall hernias. Springer, New York 2001; 487-499

17. Acevedo A, Viterbo A, Bravo J. Estudio comparativo de la infiltración con anestesia local preperitoneal con la infiltración dentro de la vaina del músculo recto en la cirugía de las hernias de la línea media. Libro de resúmenes del LVIII Congreso de la Sociedad de Cirujanos de Chile 2005. 